\title{
Successful treatment of Pseudomonas paucimobilis haemodialysis catheter-related sepsis without catheter
} removal

\author{
David Saltissi and David J. Macfarlane ${ }^{1}$
}

Departments of Renal and 'Nuclear Medicine, Royal Brisbane Hospital, Herston, Queensland 4029, Australia

\begin{abstract}
Summary: Infection is the most common complication of long-term central venous access lines. We report a case of central venous haemodialysis catheter infection with Pseudomonas paucimobilis, successfully treated by prolonged antibiotic administration without catheter removal. The implications are discussed.
\end{abstract}

\section{Introduction}

Infection of percutaneous central venous haemodialysis catheters may involve the catheter per se (catheter-related sepsis), the subcutaneous tunnel, or the superficial exit site. Many techniques have been advocated for the diagnosis of catheterrelated sepsis, but all suffer from imperfect sensitivity and specificity. Lack of uniform diagnostic criteria further complicates comparison of literature regarding prognosis and treatment. For the treatment of tunnel infections, catheter removal is generally advised. Catheter removal for catheterrelated sepsis is more controversial, particularly as it may result in significant clinical difficulties in certain circumstances.

We describe a technique of diagnosis and successful treatment of catheter-related sepsis without catheter removal in a medically complicated haemodialysis patient.

\section{Case report}

In 1986 a 69 years old female with moderate aortic stenosis and angina pectoris developed end-stage chronic renal failure due to analgesic nephropathy. This was initially treated by chronic ambulatory peritoneal dialysis (CAPD), but recurrent diverticulitis and peritonitis necessitated conversion to haemodialysis. This was performed initially via a right radio-cephalic arteriovenous fistula which thrombosed after only a few treatments. Over the next 23 months, six further arteriovenous anastomoses involving all limbs and including two

Correspondence: D. Saltissi, M.D., M.R.C.P. Accepted: 23 June 1993 prosthetic grafts also failed. Interval dialysis through short-term subclavian venous catheters was complicated by inadequate blood flow rates, difficulties with insertion and, at different times, sepsis with Streptococcus faecalis. Staphylococcus epidermidis and Klebsiella pneumoniae. During this period aminoglycoside-induced ototoxicity developed. In February 1990 a right internal jugular single-lumen silicone rubber catheter (Francis catheter, Kimal Scientific K45-1) was surgically inserted with subcutaneous tunnelling. This allowed satisfactory and uncomplicated dialysis over the next 13 months.

In April 1991 on five successive occasions immediately following dialysis the patient developed fever, nausea and frontal headache. No focus of infection was evident clinically. Repeated blood cultures drawn both centrally and peripherally were sterile. Urine culture, chest radiography and echocardiography revealed no focus of infection. Treatment with oral amoxycillin and later intravenous vancomycin was ineffective. Seventeen days after the initial episode it was decided to culture the undiluted contents of the catheter lumen specifically followed by drawing of high-volume blood cultures through the catheter prior to dialysis. The catheter contents grew Pseudomonas paucimobilis, reported as sensitive to amoxycillin, gentamicin and ticarcillin. It was postulated that the catheter was the site of recurrent bacteraemia, initiated by the dialysis procedure. In view of the previous vascular access problems the patient opposed treatment involving catheter removal despite the explained risks of antibiotic treatment failure including the development of bacterial endocarditis. It was therefore decided to give amoxycillin $1 \mathrm{~g}$ mixed with heparin 
twice daily, administered slowly through and allowed to dwell in the Francis catheter, which remained in situ. The mixture was withdrawn prior to each haemodialysis session and a fresh preparation instilled subsequently. Therapy was continued for an arbitrary period of 4 weeks. On this regimen the patient's symptoms resolved and repeated high-volume blood cultures were sterile. She has remained free of catheter-related infection to date.

\section{Discussion}

Pseudomonas paucimobilis is a water-borne opportunistic pathogen isolated widely in hospital environments. Infection may manifest as septicaemia, empyema, meningitis, abscess formation or peritonitis. Antibiotic sensitivity of the organism is variable. It may be implicated in central venous haemodialysis catheter infections. ${ }^{1}$

Central venous catheter infection is an increasing problem, with a rate of 1.37 infections per 1,000 patient catheter days in one series. ${ }^{2}$ Differential counting of micro-organism colony-forming units in peripheral and central venous blood cultures has gained greatest popularity as a diagnostic method for catheter-related sepsis not requiring catheter removal. This technique is specific but not very sensitive, especially as positive peripheral blood cultures have been reported in less than $20 \%$ of suspected cases of catheter-related infection in some series. ${ }^{3}$

Removal and re-siting of the catheter has a high cure rate. ${ }^{4}$ Nevertheless, catheter relocation may not be easy, and each insertion carries a $4-11 \% \gtreqless$ risk of major complications such as pneumothorax $\stackrel{\varnothing}{\Omega}$ or inadvertent arterial puncture. ${ }^{5}$ As an alternative, $\stackrel{\complement}{\complement}$ catheter change over a guide wire has been advocated, usually with semi-quantitative tip culture. ${ }^{6}$ Recent animal studies have documented the intravascular liberation of bacterial microemboli $\frac{\bar{\sigma}}{\bar{\rho}}$ and transfer of biofilm organisms to new catheters during such exchanges. ${ }^{7}$ The practical significance of these events is yet to be clearly defined.

In aminal models there has been little success in $\vec{\circ}$ eradicating biofilm organisms with parenteral antibiotics. However, in man, treatment of catheter $\vec{\omega}$ infections without haemodynamic compromise with antibiotics alone, leaving a catheter which is probably infected in situ actually has a reported success rate of $65-80 \%{ }^{3,8}$ This dichotomy may relate to our continued imperfect understanding of the nature of biofilm organisms.

Accordingly, in the light of this case, we suggest that in haemodynamically stable patients with $\frac{\text { ㅇ }}{3}$ suspected or proven bacterial catheter-related sep- $\overrightarrow{ }$ sis it is reasonable to initially leave the catheter in situ and start treatment with appropriate parenteral antibiotics infused slowly into and $\stackrel{\equiv}{2}$ allowed to dwell in the catheter. We believe this maintains prolonged high drug concentrations at $\varnothing$ the probable primary site of infection. The duration of therapy remains arbitrary but should probably not be less than 3 weeks. Prompt catheter removal should follow any clinical deterioration and, of course, remains the accepted primary treatment when the causative organism is a fungus. $^{2}$

\section{References}

1. Calubiran, O.V., Schoch, P.E. \& Cunha, B.A. Pseudomonas paucimobilis bacteraemia associated with haemodialysis. $J$ Hosp Infect 1990, 15: 383-388.

2. Clarke, D.E. \& Raffin, T.A. Infectious complications of indwelling long-term central venous catheters. Chest 1990, 7: 966-972.

3. Flynn, P.M., Shenep, J.L., Stokes, D.C. \& Barrett, F.F. In situ management of confirmed central venous catheter-related bacteremia. Pediatr Infect Dis J 1987, 6: 729-734.

4. Elting, L.S. \& Bodey, G.P. Septicaemia due to Xanthomas species and non-aeruginosa Pseudomonas species: increasing incidence of catheter-related infections. Medicine 1990, 69: 296-306.

5. Eyer, S., Brummitt, C., Crossley, K., Siegel, R. \& Cerra, F Catheter-related sepsis: prospective, randomized study of three methods of long-term catheter maintenance. Crit Care Med 1990, 18: 1073-1079.

6. Bozetti, F., Terno, G., Bonfanti, G. et al. Prevention and treatment of central venous catheter sepsis by exchange via a guidewire. Ann Surg 1983, 198: 48-52.

7. Olson, I.E., Lam, K., Bodey, G.P., King, E.G. \& Costerton, J.W. Evaluation of strategies for central venous cathete replacement. Crit Care Med 1992, 20: 797-804.

8. Benezra, D., Kiehn, T.E., Gold, J.W.M., Brown, A.E., Turnbull, A.D.M. \& Armstrong, D. Prospective study of infections in indwelling central venous catheters using quantitative blood cultures. Am J Med 1988, 85: 495-498. 